\title{
Plane Elastostatic Solution in an Infinite Functionally Graded Layer Weakened by a Crack Lying in the Middle of the Layer
}

\author{
R. Patra, ${ }^{1}$ S. P. Barik, ${ }^{2}$ M. Kundu, ${ }^{3}$ and P. K. Chaudhuri ${ }^{4}$ \\ ${ }^{1}$ Department of Mathematics, Hooghly Engineering \& Technology College, Hooghly, West Bengal 712 103, India \\ ${ }^{2}$ Department of Mathematics, Gobardanga Hindu College, 24 Parganas (North), West Bengal 743 273, India \\ ${ }^{3}$ Makhla Debiswari Vidyaniketan, Hooghly, West Bengal 712 245, India \\ ${ }^{4}$ Department of Applied Mathematics, University of Calcutta, 92 A. P. C. Road, Kolkata 700 009, India
}

Correspondence should be addressed to S. P. Barik; spbarik1@gmail.com

Received 17 June 2014; Revised 6 November 2014; Accepted 14 November 2014; Published 25 November 2014

Academic Editor: Sheung-Hung Poon

Copyright (C) 2014 R. Patra et al. This is an open access article distributed under the Creative Commons Attribution License, which permits unrestricted use, distribution, and reproduction in any medium, provided the original work is properly cited.

\begin{abstract}
This paper is concerned with an internal crack problem in an infinite functionally graded elastic layer. The crack is opened by an internal uniform pressure $p_{0}$ along its surface. The layer surfaces are supposed to be acted on by symmetrically applied concentrated forces of magnitude $P / 2$ with respect to the centre of the crack. The applied concentrated force may be compressive or tensile in nature. Elastic parameters $\lambda$ and $\mu$ are assumed to vary along the normal to the plane of crack. The problem is solved by using integral transform technique. The solution of the problem has been reduced to the solution of a Cauchy-type singular integral equation, which requires numerical treatment. The stress-intensity factors and the crack opening displacements are determined and the effects of graded parameters on them are shown graphically.
\end{abstract}

\section{Introduction}

The study relating the behaviour of elastic material under applied load needs special attention and care when the elastic body develops a crack in it. It is obvious that the presence of a crack in a structure not only affects the stress distribution but also drastically reduces the life span of the structure. Propagation of elastic disturbance in a solid is also disturbed by the presence of a crack. But cracks are present essentially in all structural materials, either as natural defects or as a result of fabrication processes. Stress distribution in a body which develops a crack in it is entirely different from that in a body without a crack. In literature, considerable effort has been devoted to the study of cracks in solids, due to their applications in industry in general and in fabrication of electronic components in particular.

Presence of a crack in a solid significantly affects its response to the applied load. Stress distributions in the solid with a crack are studied in two regions: the region in the neighbourhood of crack, called the near field region, and the region far away from the crack, called the far field region. Study of stress distribution in the near field region is very important. Stress-intensity factor, crack energy, and so forth are some of the quantities responsible for spreading of a crack. For a solid with a crack in it loaded mechanically or thermally, determination of stress-intensity factor (SIF) becomes a very important topic in fracture mechanics. The SIF is a parameter that gives a measure of stress concentration around cracks and defects in a solid. SIF needs to be understood if we are to design fracture tolerant materials used in bridges, buildings, aircraft, or even bells. A crack detected on the surface of a body can not be removed just by polishing. Typically for most materials whenever a crack develops in the solid, the solid is very close to the critical state of stress predicted by the SIF.

With increasingly wide application of functionally graded materials in practical engineering, the study of crack problems in functionally graded media has received much interest. Functionally graded materials (FGMs) $[1,2]$ can usually be viewed as special inhomogeneous materials whose properties vary gradually with location within the body. In FGMs, 


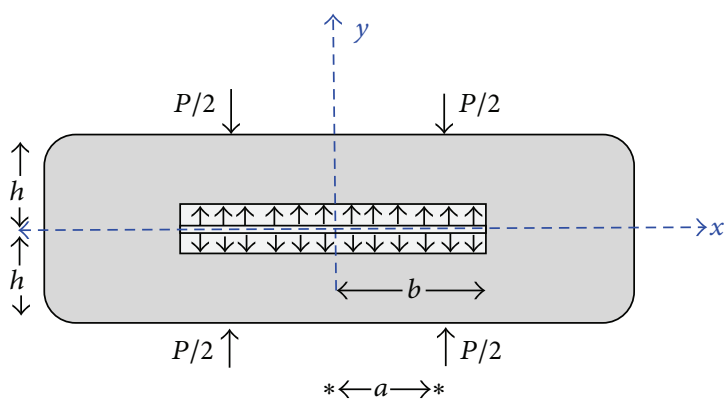

(a) Concentrated compressive load condition

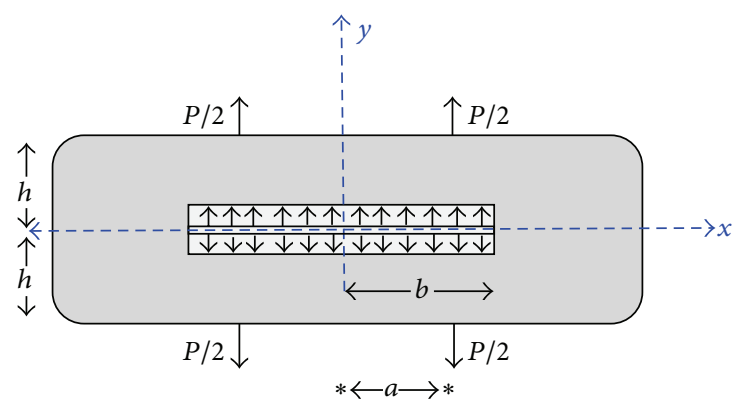

(b) Concentrated tensile load condition

FIGURE 1: Geometry of the problem.

due to the continuous change of material properties in space, the absence of interfaces between different constituents or phases largely reduces the degree of material property mismatch and brings appealing physical behaviors superior to homogeneous and conventional materials. For example, for the classic ceramic/metal FGMs, the ceramic phase offers thermal barrier effects and protects the metal from corrosion and oxidation, and the FGM is toughened and strengthened by the metallic constituent. FGMs formed by appropriately combining two or more materials in a perfectly designed manner can be more resistant to crack initiation and propagation. FGMs have potential applications in automotive brakes and clutches. FGMs offer great promise in applications where the operating conditions are severe: for example, as wear-resistant linings for handling large heavy abrasive ore particles, rocket heat shields, heat exchanger tubes, thermoelectric generators, and so forth. Because of enormous application of FGMs in industry and in various engineering designs like aerospace, submarine structures, civil engineering structures, and so forth the study of these material structures has been of increasing interest to scientists and engineers. Thus the study of solid mechanics problems should not be restricted to the isotropic and homogeneous elastic medium only, it should be extended to much more applicable fields and materials.

Crack problem in isotropic elastic medium has been extensively studied in literature following classical theory. A comprehensive list of work on crack problems by earlier investigators has been provided in Chaudhuri and Ray [3], Ozturk and Erdogan [4], Yong and Hanson [5], Fabrikant et al. [6], Fabrikant [7], Dag and Erdogan [8], Sherief and ElMaghraby [9], Birinci et al. [10], Barik et al. [11, 12], Chen et al. [13], Zhao et al. [14], Matysiak and Pauk [15], Lee [16], Gupta and Erdogan [17], and Matbuly [18].

The present investigation aims at finding the elastostatic solution in an infinite functionally graded layer weakened by an internal crack. Following the integral transform technique the problem has been reduced to a problem of Cauchy-type singular integral equation, which has been solved numerically. Numerical computations have been done to assess the effects of graded parameter considered in the problem on various subjects of interest and the results have been shown graphically.

\section{Formulation of the Problem}

We consider an infinitely long functionally graded layer of thickness $2 h$ weakened by the presence of an internal crack of length $2 b$, which is opened by a uniform internal pressure $p_{0}$ along its surface. The layer is subjected to two different types of loadings on its surfaces in a direction perpendicular to its length: (i) a symmetric pair of compressive concentrated normal loads $P / 2$ and (ii) a symmetric pair of tensile concentrated normal loads $P / 2$ (Figures $1(\mathrm{a})$ and $1(\mathrm{~b})$ ). The gravitational force has not been taken into consideration. The problem is formulated in Cartesian coordinate system $(x, y)$ in which crack lies along $x$-axis with origin at the centre of the crack. In deriving analytical solution in the present study the elastic parameters $\lambda$ and $\mu$ have been assumed to vary exponentially in the direction perpendicular to the plane of crack; that is,

$$
\lambda=\lambda_{0} e^{\beta|y|}, \quad \mu=\mu_{0} e^{\beta|y|}, \quad-h \leq y \leq h,
$$

where $\lambda_{0}$ and $\mu_{0}$ are the elastic parameters in the homogeneous medium and $\beta$ is the nonhomogeneity parameter controlling the variation of the elastic parameters in the graded medium. The strain displacement relations, linear stress-strain relations, and equations of equilibrium are, respectively, given by

$$
\begin{gathered}
\varepsilon_{x}=\frac{\partial u}{\partial x}, \quad \varepsilon_{y}=\frac{\partial v}{\partial y}, \quad \gamma_{x y}=\frac{1}{2}\left(\frac{\partial u}{\partial y}+\frac{\partial v}{\partial x}\right) \\
\sigma_{x}=\frac{\mu}{\kappa-1}\left[(1+\kappa) \varepsilon_{x}+(3-\kappa) \varepsilon_{y}\right] \\
\sigma_{y}=\frac{\mu}{\kappa-1}\left[(3-\kappa) \varepsilon_{x}+(1+\kappa) \varepsilon_{y}\right] \\
\tau_{x y}=2 \mu \gamma_{x y} \\
\frac{\partial \sigma_{x}}{\partial x}+\frac{\partial \tau_{x y}}{\partial y}=0 \\
\frac{\partial \tau_{x y}}{\partial x}+\frac{\partial \sigma_{y}}{\partial y}=0
\end{gathered}
$$


where $\kappa=3-4 \nu$ and $\nu$ is Poisson's ratio. The present problem is equivalent to the following mathematical problem with the following.

(i) Equilibrium Equations. Consider

$$
\begin{gathered}
2(1-v) \frac{\partial^{2} u}{\partial x^{2}}+(1-2 v) \frac{\partial^{2} u}{\partial y^{2}}+\frac{\partial^{2} v}{\partial x \partial y} \\
+\beta(1-2 v)\left(\frac{\partial u}{\partial y}+\frac{\partial v}{\partial x}\right)=0, \\
(1-2 v) \frac{\partial^{2} v}{\partial x^{2}}+2(1-v) \frac{\partial^{2} v}{\partial y^{2}}+\frac{\partial^{2} u}{\partial x \partial y} \\
+\beta\left[2(1-v) \frac{\partial v}{\partial y}+2 v \frac{\partial u}{\partial x}\right]=0 .
\end{gathered}
$$

(ii) The Boundary Conditions. Consider

$$
\begin{gathered}
\tau_{x y}(x, 0)=0, \quad(-\infty<x<\infty), \\
\tau_{x y}(x, h)=0, \quad(-\infty<x<\infty), \\
\sigma_{y}(x, h)=\mp\left[\frac{P}{2} \delta(x-a)+\frac{P}{2} \delta(x+a)\right], \\
\quad(-\infty<x<\infty), \\
\frac{\partial}{\partial x}[v(x, 0)]= \begin{cases}f(x), & |x|<b ; \\
0, & |x|>b .\end{cases} \\
\sigma_{y}(x, 0)=-p_{0}, \quad(-b \leq x \leq b),
\end{gathered}
$$

where $u$ and $v$ are the $x$ and $y$ components of the displacement vector; $\sigma_{x}, \sigma_{y}, \tau_{x y}$ are the normal and sharing stress components; $f(x)$ is an unknown function; and $\delta(x)$ is the Dirac delta function. In (9) positive sign indicates tensile force while negative sign corresponds to compressive force.

\section{Method of Solution}

First of all we observe that, due to symmetry of the crack location with respect to the layer, nature of the graded parameter and also of the applied load with respect to the crack, it is sufficient to consider solution of the problem in the regions $0 \leq x<\infty$ and $0 \leq y \leq h$. To solve the partial differential equations (6), the Fourier transform is applied to the equations with respect to the variable $x$.

Utilizing the symmetry condition the displacement components $u, v$ may be written as

$$
\begin{aligned}
& u(x, y)=\frac{2}{\pi} \int_{0}^{\infty} \Phi(\alpha, y) \sin (\alpha x) d \alpha, \\
& v(x, y)=\frac{2}{\pi} \int_{0}^{\infty} \Psi(\alpha, y) \cos (\alpha x) d \alpha,
\end{aligned}
$$

where $\Phi(\alpha, y)$ and $\Psi(\alpha, y)$ are Fourier transforms of $u(x, y)$ and $v(x, y)$, respectively, with respect to the coordinate $x$ and $\alpha$ is the transformed parameter.
Substituting $u(x, y)$ and $v(x, y)$ from (12) into the equations of equilibrium (6) we obtain the differential equation for the determination of $\Phi(\alpha, y)$

$$
\begin{aligned}
\Phi^{i v}+ & 2 \beta \Phi^{\prime \prime \prime}-\left(2 \alpha^{2}-\beta^{2}\right) \Phi^{\prime \prime} \\
& -2 \alpha^{2} \beta \Phi^{\prime}+\alpha^{2}\left\{\alpha^{2}-\frac{(\kappa-3)}{\kappa+1} \beta^{2}\right\} \Phi=0 .
\end{aligned}
$$

The solution of this equation is of the form

$$
\Phi(\alpha, y)=\sum_{i=1}^{4} A_{i}(\alpha) e^{m_{i} y}
$$

where $A_{i}(\alpha),(i=1, \ldots, 4)$ are constants to be determined from the boundary conditions and $m_{1}, m_{2}, m_{3}, m_{4}$ are the four complex roots of two biquadratic equation

$$
\begin{aligned}
m^{4}+ & 2 \beta m^{3}-\left(2 \alpha^{2}-\beta^{2}\right) m^{2} \\
& -2 \alpha^{2} \beta m+\alpha^{2}\left\{\alpha^{2}-\frac{\kappa-3}{\kappa+1} \beta^{2}\right\}=0
\end{aligned}
$$

The function $\Psi(\alpha, y)$ can then be determined as

$$
\Psi(\alpha, y)=\sum_{i=1}^{4} M_{i}(\alpha) A_{i}(\alpha) e^{m_{i} y}
$$

It follows from (15) that

$$
\begin{aligned}
& m_{1}=\bar{m}_{3}=-\frac{\beta}{2}+\sqrt{\alpha^{2}+\frac{\beta^{2}}{4}+i \alpha \beta \sqrt{\frac{3-\kappa}{\kappa+1}}}, \\
& m_{2}=\bar{m}_{4}=-\frac{\beta}{2}-\sqrt{\alpha^{2}+\frac{\beta^{2}}{4}+i \alpha \beta \sqrt{\frac{3-\kappa}{\kappa+1}}},
\end{aligned}
$$

where $\bar{m}_{3}, \bar{m}_{4}$ denote the respective complex conjugates of $m_{1}, m_{2}$. In (16), the known functions $M_{i}(\alpha)(i=1, \ldots, 4)$ may be expressed as follows:

$$
\begin{array}{r}
M_{i}(\alpha)=\frac{(\kappa-1) m_{i}^{2}+\beta(\kappa-1) m_{i}-\alpha^{2}(\kappa+1)}{\alpha\left\{2 m_{i}+\beta(\kappa-1)\right\}}, \\
(i=1, \ldots, 4) .
\end{array}
$$


Substituting (12) into (2) and (3) and utilizing (14) and (16) we obtain

$$
\begin{aligned}
& \frac{1}{2 \mu} \sigma_{x}(x, y) \\
&=\frac{2}{\pi} \int_{0}^{\infty} \sum_{i=1}^{4} \frac{1}{2(1-\kappa)}\left[-(1+\kappa) \alpha-(3-\kappa) m_{i} M_{i}\right] \\
& \times A_{i} e^{m_{i} y} \cos (\alpha x) d \alpha
\end{aligned}
$$$$
\frac{1}{2 \mu} \sigma_{y}(x, y)
$$$$
=\frac{2}{\pi} \int_{0}^{\infty} \sum_{i=1}^{4} \frac{1}{2(1-\kappa)}\left[-(1+\kappa) m_{i} M_{i}-(3-\kappa) \alpha\right]
$$$$
\times A_{i} e^{m_{i} y} \cos (\alpha x) d \alpha,
$$$$
\frac{1}{2 \mu} \tau_{x y}(x, y)
$$$$
=\frac{2}{\pi} \int_{0}^{\infty} \sum_{i=1}^{4}\left[-\frac{\alpha}{2} M_{i}+\frac{m_{i}}{2}\right] A_{i} e^{m_{i} y} \sin (\alpha x) d \alpha .
$$

From the boundary conditions (7)-(10), the unknown constants $A_{i}(i=1,2, \ldots, 4)$ can be found out from the following linear algebraic system of equations:

$$
\begin{gathered}
{\left[\begin{array}{cccc}
S_{1} e^{m_{1} h} & S_{2} e^{m_{2} h} & S_{3} e^{m_{3} h} & S_{4} e^{m_{4} h} \\
T_{1} e^{m_{1} h} & T_{2} e^{m_{2} h} & T_{3} e^{m_{3} h} & T_{4} e^{m_{4} h} \\
m_{1}-T_{1} & m_{2}-T_{2} & m_{3}-T_{3} & m_{4}-T_{4} \\
T_{1} & T_{2} & T_{3} & T_{4}
\end{array}\right]\left[\begin{array}{c}
A_{1} \\
A_{2} \\
A_{3} \\
A_{4}
\end{array}\right]} \\
=\left[\begin{array}{c}
-R_{1}(\alpha) \\
0 \\
-R_{2}(\alpha) \\
0
\end{array}\right],
\end{gathered}
$$

where

$$
\begin{gathered}
R_{1}(\alpha)= \pm \frac{(\kappa-1)}{2 \mu} P \cos (a \alpha), \\
R_{2}(\alpha)=\int_{0}^{\infty} f(x) \sin (\alpha x) d x, \\
S_{i}=(1+\kappa) m_{i} M_{i}+(3-\kappa) \alpha, \\
T_{i}=-\alpha M_{i}+m_{i}, \quad(i=1, \ldots, 4) .
\end{gathered}
$$

Equation (22) yields

$$
A_{i}(\alpha)=(-1)^{i}\left[\frac{D_{1 i}}{D} R_{1}(\alpha)+\frac{D_{3 i}}{D} R_{2}(\alpha)\right], \quad(i=1, \ldots, 4),
$$

where $D$ is the determinant and $D_{j i}(j=1,3 ; i=1, \ldots, 4)$ is the subdeterminant (corresponding to the elimination of the $j$ th row and $i$ th column) of the coefficient matrix in (22).
Substituting the values of $A_{i}(\alpha)$ into (20) and utilizing the boundary condition (11) we get the following singular integral equation:

$$
\begin{aligned}
& \frac{1}{\pi} \int_{-b}^{b} f(t)\left[\frac{1}{t-x}+k_{1}(x, t)\right] d t \\
& \quad=\frac{(\kappa-1)}{\mu \chi}\left[-p_{0} \pm \frac{P}{2 \pi} k_{2}(x)\right], \quad(-b<x<b),
\end{aligned}
$$

where

$$
\begin{gathered}
k_{1}(x, t)=\frac{1}{\chi} \int_{0}^{\infty}\left\{\sum_{i=1}^{4}(-1)^{i} \frac{D_{3 i}}{D} S_{i}-\chi\right\}, \\
\chi=\lim _{\alpha \rightarrow \infty} \sum_{i=1}^{4}(-1)^{i} \frac{D_{3 i}}{D} S_{i}, \\
k_{2}(x)=\int_{0}^{\infty} \sum_{i=1}^{4}(-1)^{i} \frac{D_{1 i}}{D} S_{i} \\
\quad \times\{\cos \alpha(x-a)+\cos \alpha(a+x)\} d \alpha .
\end{gathered}
$$

The kernels $k_{1}(x, t)$ and $k_{2}(x)$ are bounded and continuous in the closed interval $-b \leq x \leq b$. The integral equation (25) must be solved under the following single-valuedness condition:

$$
\int_{-b}^{b} f(t) d t=0
$$

Before further proceeding it will be convenient to introduce nondimensional variables $r$ and $s$ by rescaling all lengths in the problems by length scale $b$ :

$$
\begin{gathered}
x=b r, \quad t=b s, \\
f(t)=f(b s)=\frac{p_{0}(1-\kappa)}{\mu(3-\kappa)} \phi(s), \quad \omega=\alpha b .
\end{gathered}
$$

In terms of nondimensional variables the integral equation (25) and single valuedness condition (27) become

$$
\begin{gathered}
\frac{1}{\pi} \int_{-1}^{1}\left[\frac{1}{s-r}+k_{1}^{*}(r, s)\right] \phi(s) d s \\
=-1 \pm \frac{Q}{\pi} k_{2}^{*}(r), \quad(-1<r<1), \\
\int_{-1}^{1} \phi(s) d s=0,
\end{gathered}
$$

where

$$
\begin{aligned}
& k_{1}^{*}(r, s)=\frac{1}{\chi} \int_{0}^{\infty}\left\{\sum_{i=1}^{4}(-1)^{i} \frac{D_{3 i}}{D} S_{i}-\chi\right\} \sin \omega(s-r) d \omega, \\
& k_{2}^{*}(r)=\int_{0}^{\infty} \sum_{i=1}^{4}(-1)^{i} \frac{D_{1 i}}{D} S_{i} \\
& \quad \times\left\{\cos \omega\left(r-a^{*}\right)+\cos \omega\left(r+a^{*}\right)\right\} d \omega,
\end{aligned}
$$


$a^{*}=a / b$, and $Q$ is the load ratio defined as

$$
Q=\frac{P}{2 b p_{0}} .
$$

\section{Solution of Integral Equations}

The singular integral equation (30) is a Cauchy-type singular integral equation for an unknown function $\phi(s)$. For the evaluation of displacement and stress components it is necessary to solve the integral equation (30) for the unknown function $\phi(s)$. Expressing now the solution of (30) in the form

$$
\phi(s)=\frac{\psi(s)}{\sqrt{1-s^{2}}}, \quad(-1<s<1),
$$

where $\psi(s)$ is a regular and bounded unknown function and using the Gauss-Chebyshev formula (Erdogan and Gupta [19]) to evaluate the integral equation (30), we obtain

$$
\begin{aligned}
\frac{1}{N}\left[\sum_{k=1}^{N}\left\{\frac{1}{s_{k}-r_{i}}+k_{1}^{*}\left(r_{i}, s_{k}\right)\right\} \psi\left(s_{k}\right)\right] & =-1 \pm \frac{Q}{\pi} k_{2}^{*}\left(r_{i}\right), \\
i & =1,2, \ldots, N-1, \\
\frac{\pi}{N} \sum_{k=1}^{N} \psi\left(s_{k}\right) & =0,
\end{aligned}
$$

where $s_{k}$ and $r_{i}$ are given by

$$
\begin{gathered}
s_{k}=\cos \left(\frac{2 k-1}{2 N} \pi\right), \quad(k=1,2,3, \ldots, N), \\
r_{i}=\cos \left(\frac{\pi i}{N}\right), \quad(i=1,2,3, \ldots, N-1) .
\end{gathered}
$$

We observe that, corresponding to $(N-1)$ collocation points $x_{j}=\cos (j \pi / 2(N+1)), j=1,2, \ldots,(N-1),(35)$ represent a set of $N$ linear equations in $N$ unknowns $\psi\left(s_{1}\right), \psi\left(s_{2}\right), \ldots$, $\psi\left(s_{N}\right)$. This linear algebraic system of equations is solved numerically by utilizing Gaussian elimination method.

\section{Determination of Stress-Intensity Factor}

Presence of a crack in a solid significantly affects the stress distribution compared to that when there is no crack. While the stress distribution in a solid with a crack in the region far away from the crack is not much disturbed, the stresses in the neighbourhood of the crack tip assume a very high magnitude. In order to predict whether the crack has a tendency to expand further, the stress-intensity factor (SIF), a quantity of physical interest, has been defined in fracture mechanics. The load at which failure occurs is referred to as the fracture strength. The stress-intensity factor is defined as

$$
k(b)=\lim _{r \rightarrow 1} \sqrt{2 b(r-1)} \sigma_{y}^{*}(r, 0),
$$

where $\sigma_{y}^{*}(r, 0)$ is normal stress in terms of nondimensional variables.
Using (29) and (25) and after some manipulation, the expression for $k(b)$ is obtained as

$$
\frac{k(b)}{p_{0} \sqrt{b}}=-\psi(1),
$$

where $\psi(1)$ can be found out from $\psi\left(s_{k}\right)(k=1,2,3, \ldots, N)$ using the interpolation formulas given by Krenk [20].

Following the method as in Gupta and Erdogan [17] we obtain the crack surface displacement in the form

$$
v(x, 0)=\int_{-b}^{x} f(t) d t, \quad(-b<x<b) .
$$

Using (28) and (29) into (39), we can express the dimensionless normal displacement as

$$
\begin{aligned}
v^{\prime}(r, 0) & =\frac{v(x, 0) \mu(3-\kappa)}{p_{0} b(1-\kappa)} \\
& =\int_{-1}^{r} \phi(s) d s, \quad(-1<r<1),
\end{aligned}
$$

which can be obtained numerically, using Simpson's 1/3 integration formula and the appropriate interpolation formula.

\section{Numerical Results and Discussions}

The present study is related to the study of an internal crack problem in an infinite functionally graded elastic layer. The main objective of the present discussion is to study the effects of graded parameters as well as of different applied loads on stress-intensity factor and crack opening displacement. The elastic moduli $\lambda$ and $\mu$ have been assumed to be position dependent ((1) in Section 2). The presence of the graded parameters $\beta$ in FGMs makes the governing differential equations more complex to get a complete analytical solution. Solution of the problem can be obtained using numerical methods. Following the standard numerical method described in Section 4, the normal displacement component and the stress-intensity factor are computed and shown graphically.

The variation of normalized stress-intensity factor (NSIF) $k^{\prime}(b)$ with crack length $b / h$ is shown in Figure 2 for both the cases of two symmetric transverse pairs of compressive and tensile concentrated forces. It is observed from Figure 2(a) that for compressive concentrated forces the NSIF decreases with the increase of the load ratio $Q$, and the increase of $k^{\prime}(b)$ is quite significant for smaller values of $Q$. It is also observed from Figure 2(a) that the load ratio $Q$ is not of much effect on $k^{\prime}(b)$ when the crack length is sufficiently small. Contrary to this, in Figure 2(b), where the force is of tensile nature, $k^{\prime}(b)$ increases with $Q$. For small crack length, the behaviour of $k^{\prime}(b)$ is similar to the case of compressive concentrated load. In Figure 3 NSIF experiences the effect of graded parameter $\beta$ for fixed load ratio $Q$. It is observed that in both compressive and tensile load conditions $k^{\prime}(b)$ increases with graded parameter $\beta$ near the centre of the crack, while the effect is opposite far away from the centre of the crack. Figure 4 displays the variation of $k^{\prime}(b)$ for different 


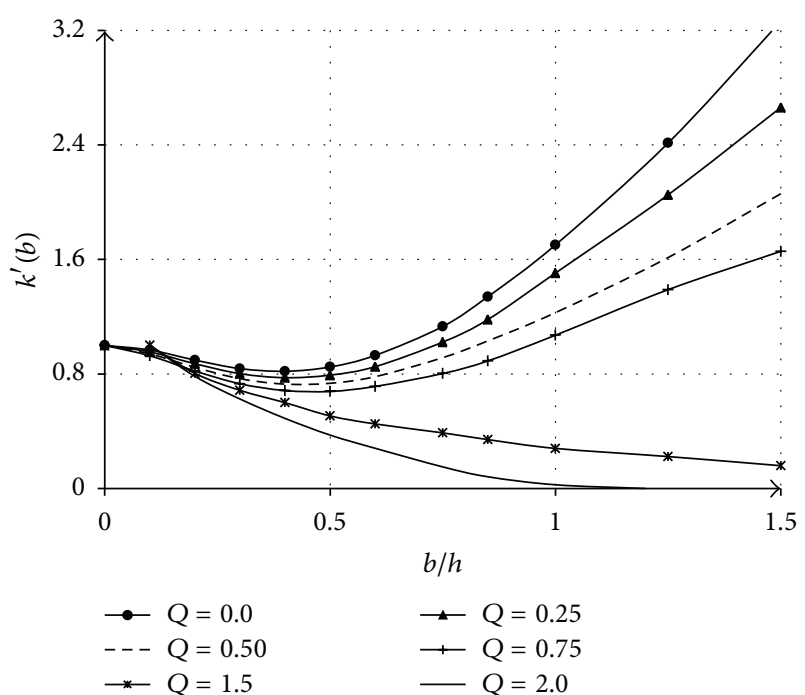

(a)

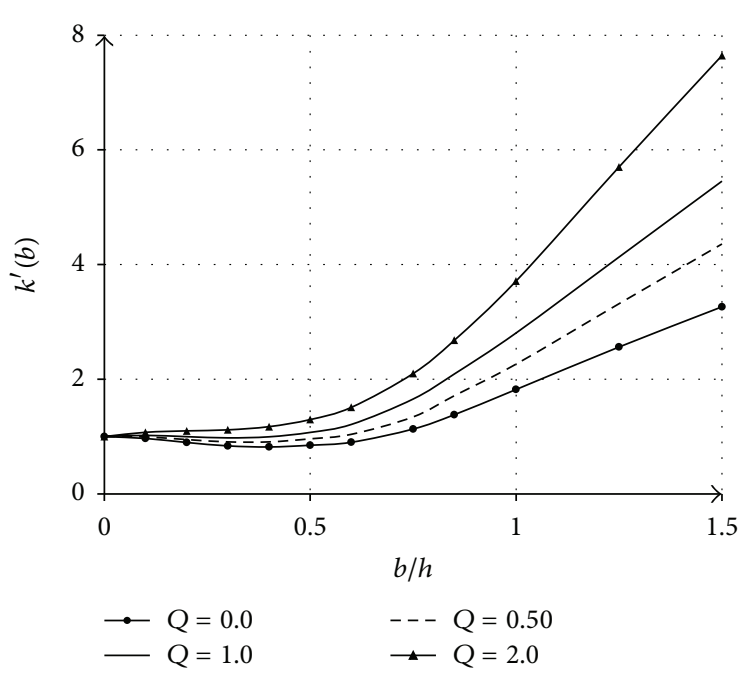

(b)

FIGURE 2: (a) Variation of normalized stress-intensity factor $k^{\prime}(b)$ with $b / h$ for different loads $Q$ in the case of compressive concentrated forces $(a / b=0.0, K=1.8$, and $\beta=0.1)$. (b) Variation of normalized stress-intensity factor $k^{\prime}(b)$ with $b / h$ for different loads $Q$ in the case of tensile forces $(a / b=0.5, K=1.8$, and $\beta=0.1)$.

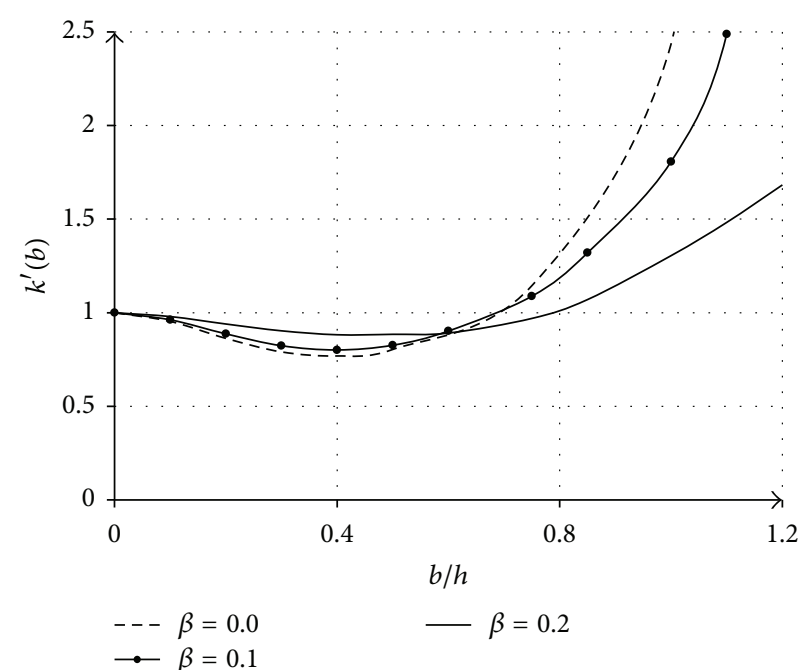

(a)

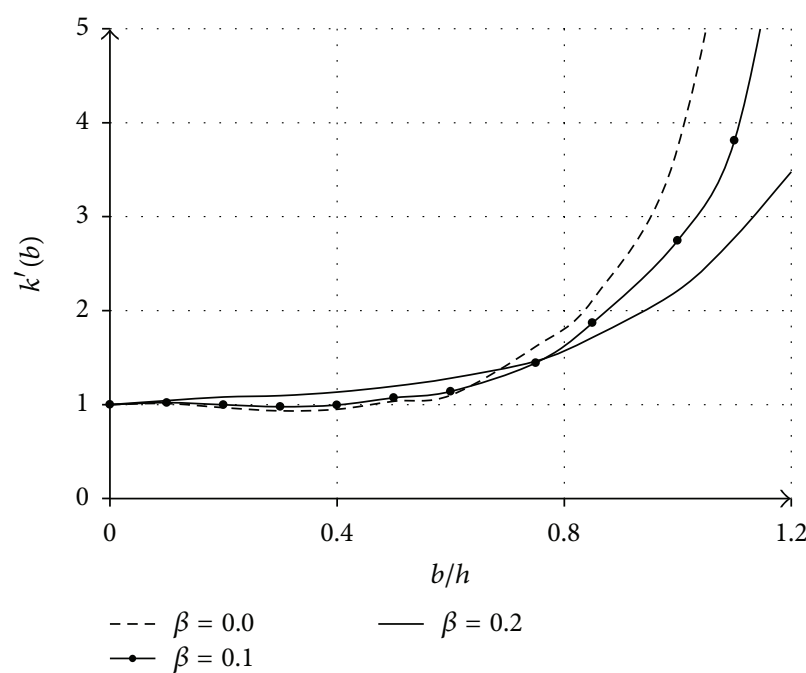

(b)

FIGURE 3: (a) Effect of graded parameter $\beta$ on normalized stress-intensity factor $k^{\prime}(b)$ in the case of compressive forces $(a / b=0.0, Q=0.1$, and $K=1.8$ ). (b) Effect of graded parameter $\beta$ on normalized stress-intensity factor $k^{\prime}(b)$ in the case of tensile forces $(a / b=0.5, Q=1.0$, and $K=1.8$ ).

position of loading. It is noted that, in the case of compressive concentrated forces, $k^{\prime}(b)$ increases with increasing $a / b$, but it decreases in the case of tensile concentrated forces.

Figure 5 depicts the variation of normalized crack surface displacement $v^{\prime}(r, 0)$ with $r / h$ for different values of load ratio $Q$. It is clear from Figure 5(a) that for compressive nature of forces $v^{\prime}(r, 0)$ decreases as load ratio $Q$ increases but decreases as load ratio $Q$ also decreases (Figure 5(b)). For both the cases of compressive and tensile concentrated forces the graphs show that the normalized crack surface displacement is symmetrical with respect to origin. The effect of graded parameter $\beta$ on $v^{\prime}(r, 0)$ is observed in Figure 6 for both the cases of compressive and tensile concentrated forces. Figure 7 illustrates the role of the point of application of loading on the normalized crack surface displacement for a particular load ratio $Q=0.1$ and $b / h=1.0$. It is observed in Figure 7(a) that for compressive concentrated loading the normalized crack surface displacement increases with the increased values of $a / b$ but behaviour is just opposite (Figure 7(b)) for tensile concentrated loading. 


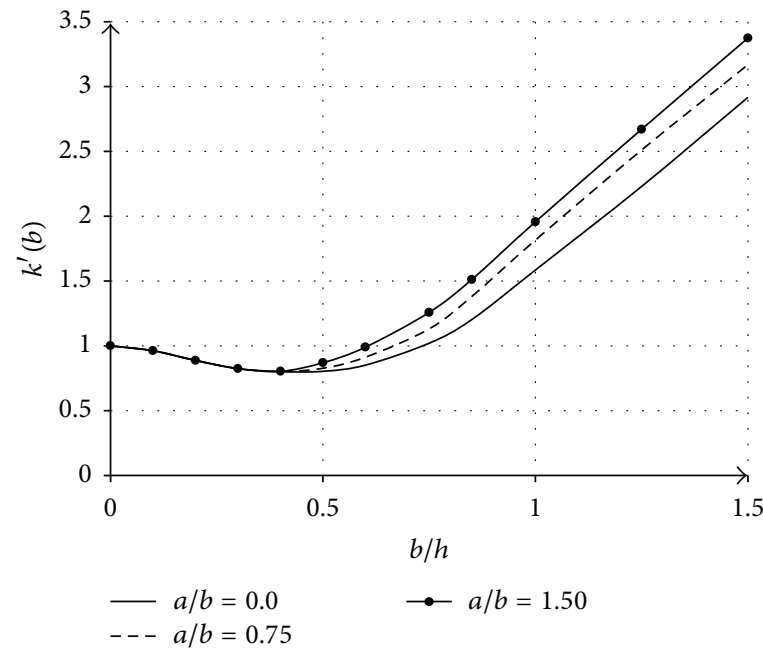

(a)

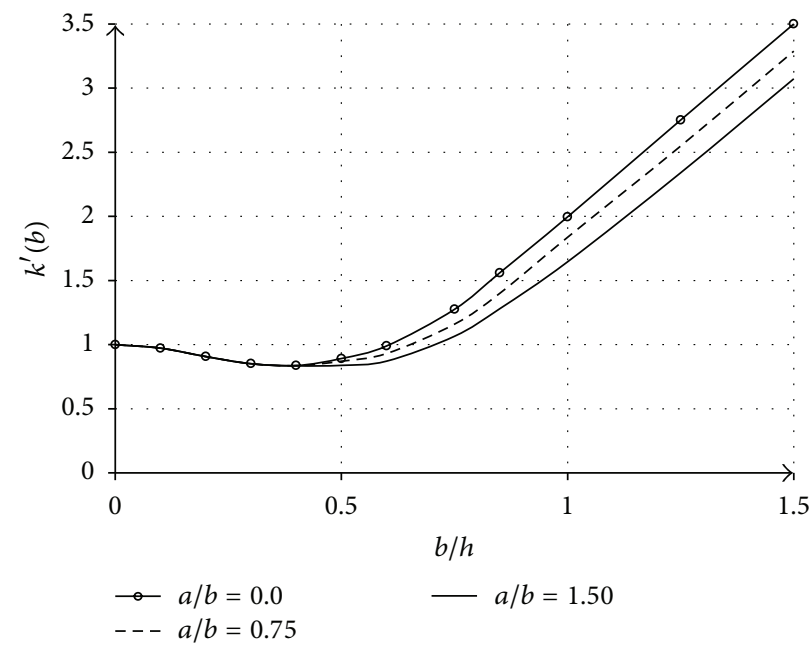

(b)

FIGURE 4: (a) Variation of normalized stress-intensity factor $k^{\prime}(b)$ for different values $a / b$ in the case of compressive forces $(Q=0.10, K=1.8$, and $\beta=0.1$ ). (b) Variation of normalized stress-intensity factor $k^{\prime}(b)$ for different values of $a / b$ in the case of tensile forces $(Q=0.1, K=1.8$, and $\beta=0.1$.

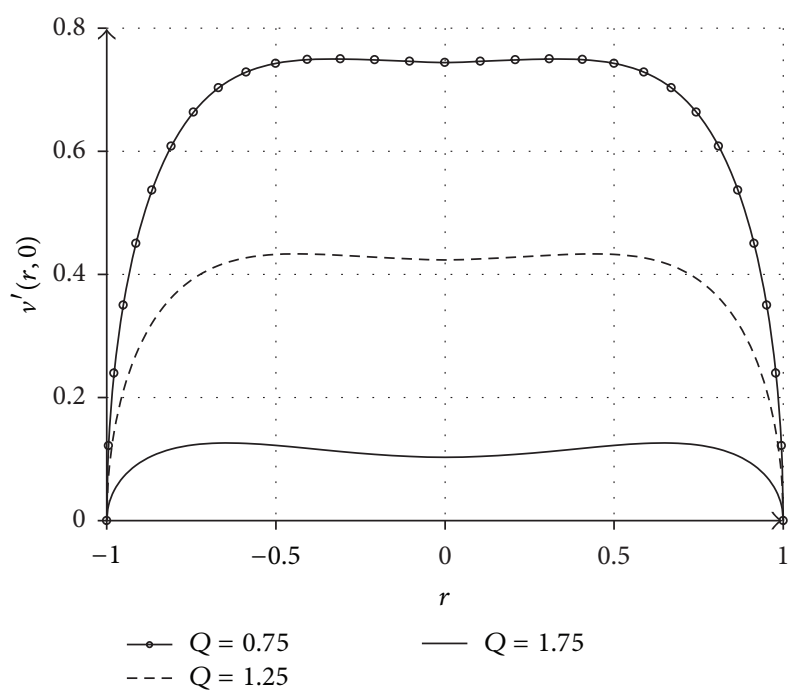

(a)

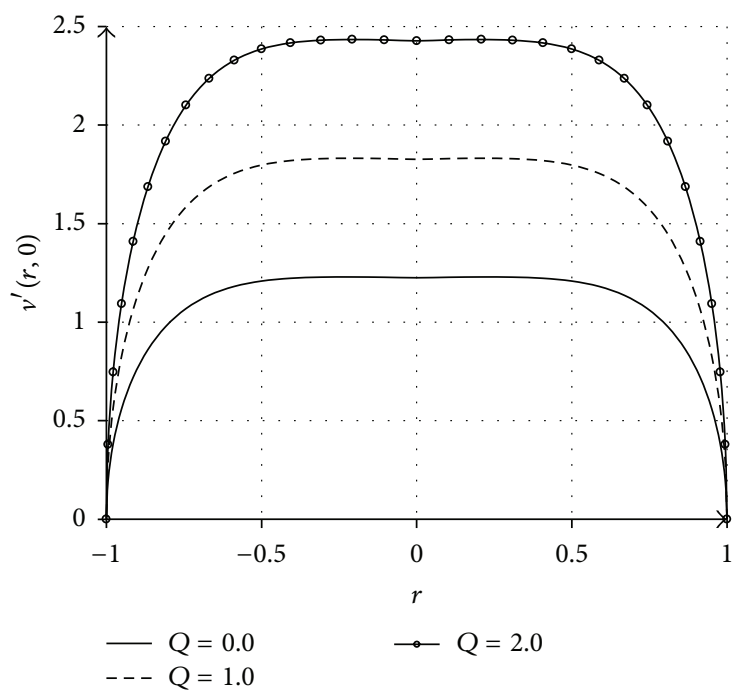

(b)

FIGURE 5: (a) Variation of normalized crack surface displacement $v^{\prime}(r, 0)$ for different load ratios $Q$ in the case of compressive forces $(a / b=0.0$, $b / h=1.0, K=1.8$, and $\beta=0.1$ ). (b) Variation of normalized crack surface displacement $v^{\prime}(r, 0)$ for different load ratios $Q$ in the case of tensile forces $(a / b=0.5, b / h=1.0, K=1.8$, and $\beta=0.1)$.

An important observation may be available from Figure 2(a), under the compressive load condition. We observe that, in this case, the layer with a crack in it is under two types of loadings. The compressive load of total magnitude $P$ at the surfaces and a force of magnitude $p_{0}$ uniformly distributed on the crack surfaces and oppositely directed to the load $P$. Clearly, the load ratio $Q=P / 2 b p_{0}$ having its value $>1$ indicates that compressive load is greater than the crack opening load. This physically means that there is very little chance of crack expansion for sufficiently large value of $Q$. In other words, the SIF at the crack tip will be zero for some value of $Q$ and also for some crack length. The corresponding crack length for that value of $Q$ represents the critical crack length. From Figure 2(a), if $Q=2.0$, the critical crack length is approximately 1.1.

\section{General Conclusion}

The present discussion relating to the study of the behavior of a layer of functionally graded material with a crack in it and under the action of compressive or tensile forces on its surfaces provides the following observations. 


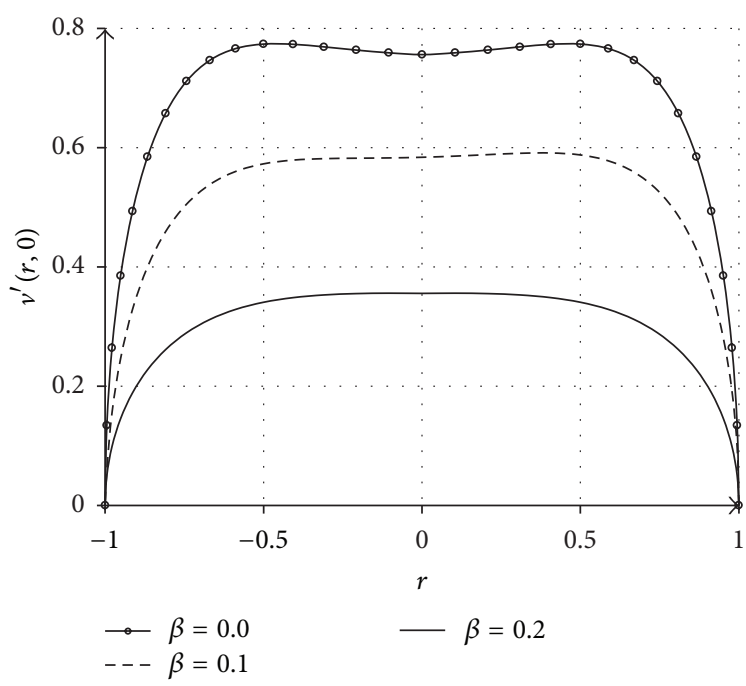

(a)

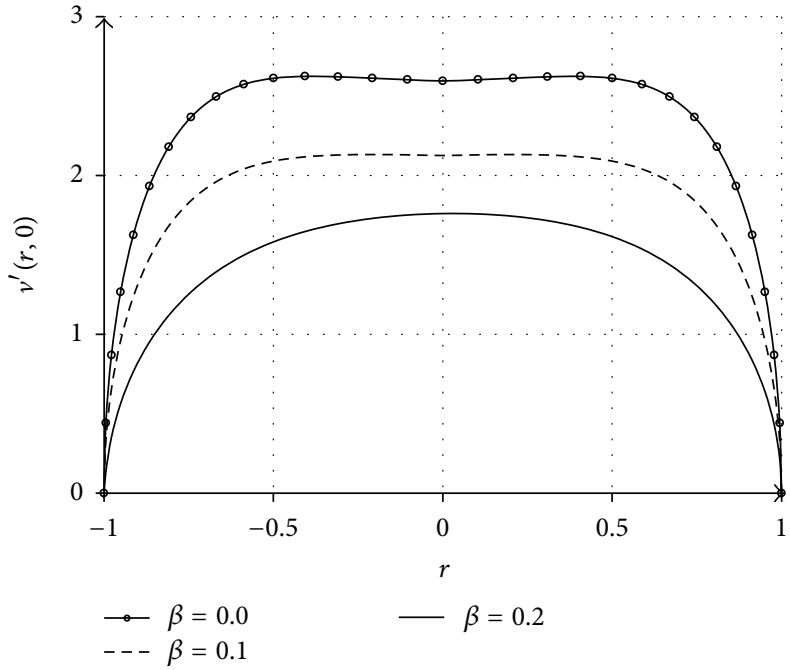

(b)

FIGURE 6: (a) Effect of graded parameter $\beta$ on normalized crack surface displacement $v^{\prime}(r, 0)$ in the case of compressive forces $(a / b=0.0$, $Q=1.0, K=1.8$, and $b / h=1.0)$. (b) Effect of graded parameter $\beta$ on normalized crack surface displacement $v^{\prime}(r, 0)$ in the case of tensile forces $(a / b=0.5, Q=1.5, K=1.8$, and $b / h=1.0)$.

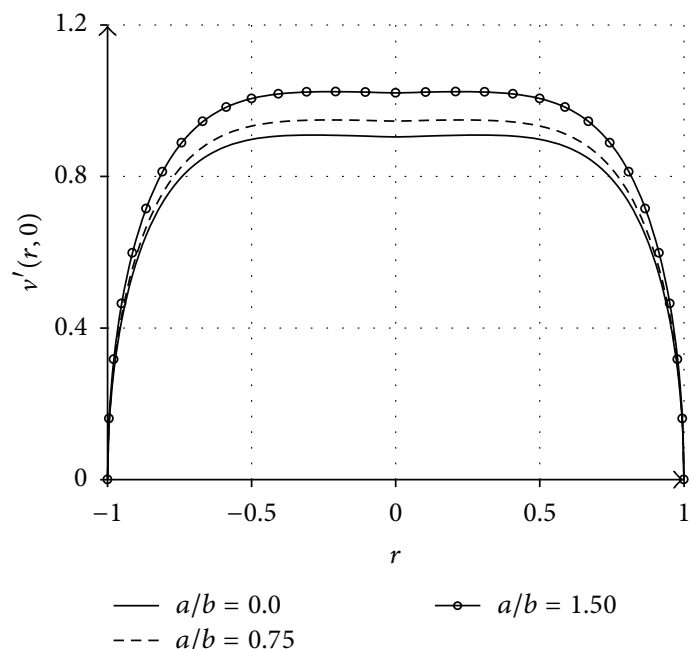

(a)

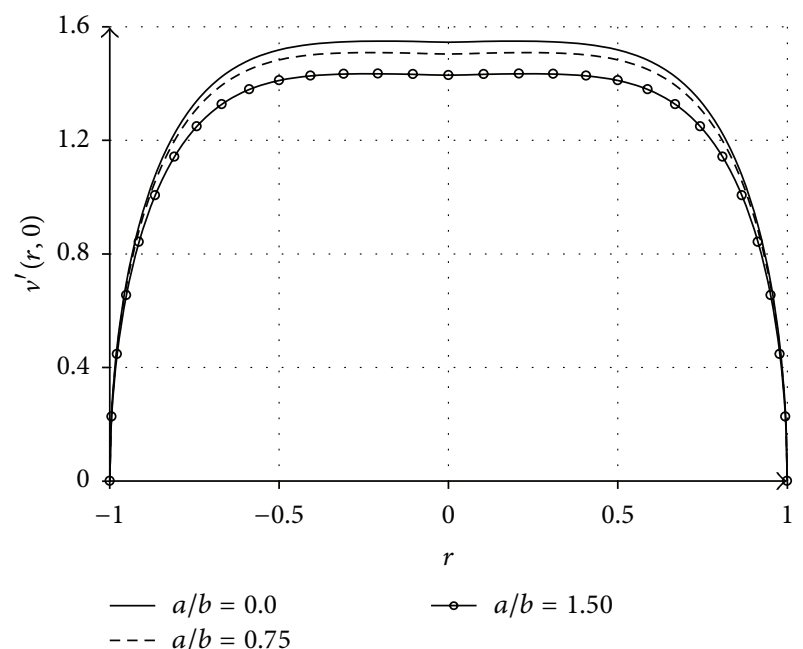

(b)

FIGURE 7: (a) Variation of normalized crack surface displacement $v^{\prime}(r, 0)$ for various values of $a / b$ in the case of compressive forces $(Q=0.1$, $K=1.8, \beta=0.1$, and $b / h=1.0)$. (b) Variation of normalized crack surface displacement $v^{\prime}(r, 0)$ for various values of $a / b$ in the case of tensile forces $(Q=0.1, K=1.8, \beta=0.1$, and $b / h=1)$.

The behavioral patterns in homogeneous and nonhomogeneous materials of the type depicted in (1) have almost been of the same nature, the only difference being in their magnitudes and variations in slopes. The SIF becomes zero for a particular type of compressive loading yielding a critical crack length. This observation is expected from physical point of view.

\section{Conflict of Interests}

The authors declare that there is no conflict of interests regarding the publication of this paper.

\section{Acknowledgments}

One of the authors, Sakti Pada Barik, is thankful to University Grants Commission, New Delhi, for providing financial assistance under the minor research project scheme. The authors also sincerely thank the reviewers for their valuable comments.

\section{References}

[1] I. Reynolds and J. Nathan, Functionally Graded Materials, Nova Science, New York, NY, USA, 2012. 
[2] Y. Miyamoto, Functionally Graded Materials: Design, Processing, and Applications, Chapman \& Hall, 1999.

[3] P. K. Chaudhuri and S. Ray, "Sudden twisting of a pennyshaped crack in a nonho mogeneous elastic medium," Journal of Mathematical and Physical Sciences, vol. 29, no. 5, pp. 207221, 1995.

[4] M. Ozturk and F. Erdogan, "The axisymmetric crack problem in a nonhomogeneous medium," Journal of Applied Mechanics, vol. 60, no. 2, pp. 406-413, 1993.

[5] Z. Yong and M. T. Hanson, "Circular crack system in an infinite elastic medium under arbitrary normal loads," Journal of Applied Mechanics, vol. 61, no. 3, pp. 582-588, 1994.

[6] V. I. Fabrikant, B. S. Rubin, and E. N. Karapetian, "External circular crack under normal load: a complete solution," Journal of Applied Mechanics, vol. 61, no. 4, pp. 809-814, 1994.

[7] V. I. Fabrikant, "Interaction of an arbitrary force with a flexible punch or with an external circular crack," International Journal of Engineering Science, vol. 34, no. 15, pp. 1753-1765, 1996.

[8] S. Dag and F. Erdogan, "A surface crack in a graded medium under general loading conditions," Journal of Applied Mechanics, vol. 69, no. 5, pp. 580-588, 2002.

[9] H. H. Sherief and N. M. El-Maghraby, "An internal pennyshaped crack in an infinite thermoelastic solid," Journal of Thermal Stresses, vol. 26, no. 4, pp. 333-352, 2003.

[10] A. Birinci, F. Birinci, F. L. Cakiroglu, and R. Erdol, "An internal crack problem for an infinite elastic layer," Archive of Applied Mechanics, vol. 80, no. 9, pp. 997-1005, 2010.

[11] S. P. Barik, M. Kanoria, and P. K. Chaudhuri, "Stress distribution in the neighbourhood of an external crack in a transversely isotropic medium," Indian Journal of Theoretical Physics, vol. 55, no. 1, pp. 85-96, 2007.

[12] S. P. Barik, M. Kanoria, and P. K. Chaudhuri, "Steady state thermoelastic problem in an in fi nite Functionally graded solid with a crack," International Journal of Applied Mathematics and Mechanics, vol. 19, pp. 44-66, 2010.

[13] W. Q. Chen, H. J. Ding, and D. S. Ling, "Thermoelastic field of a transversely isotropic elastic medium containing a penny-shaped crack: exact fundamental solution," International Journal of Solids and Structures, vol. 41, no. 1, pp. 69-83, 2004.

[14] Z. G. Zhao, X. W. Zhang, and Y. Y. Bai, "Investigation of two Griffith cracks subject to uniform tension by using the non-local theory," International Journal of Engineering Science, vol. 37, no. 13, pp. 1709-1722, 1999.

[15] S. J. Matysiak and V. J. Pauk, "On crack problem in an elastic ponderable layer," International Journal of Fracture, vol. 96, no. 4, pp. 371-380, 1999.

[16] D.-S. Lee, "The problem of internal cracks in an infinite strip having a circular hole," Acta Mechanica, vol. 169, no. 1-4, pp. 101-110, 2004.

[17] G. D. Gupta and F. Erdogan, "The problem of edge cracks in an infinite strip," Journal of Applied Mechanics, vol. 41, no. 4, pp. 1001-1006, 1974.

[18] M. S. Matbuly, "Analytical solution for an interfacial crack subjected to dynamic anti-plane shear loading," Acta Mechanica, vol. 184, no. 1-4, pp. 77-85, 2006.

[19] F. Erdogan and G. D. Gupta, "On the numerical solution of singular integral equations," Quarterly of Applied Mathematics, vol. 29, pp. 525-534, 1972.

[20] S. Krenk, "A note on the use of the interpolation polynomial for solution of singular integral equations," Quarterly of Applied Mathematics, vol. 32, pp. 479-485, 1975. 


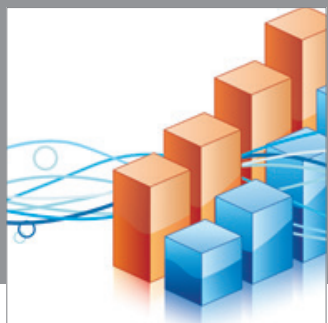

Advances in

Operations Research

mansans

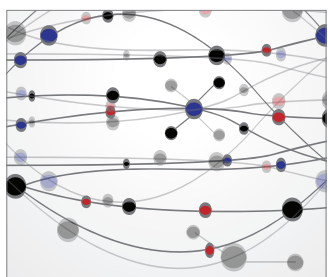

The Scientific World Journal
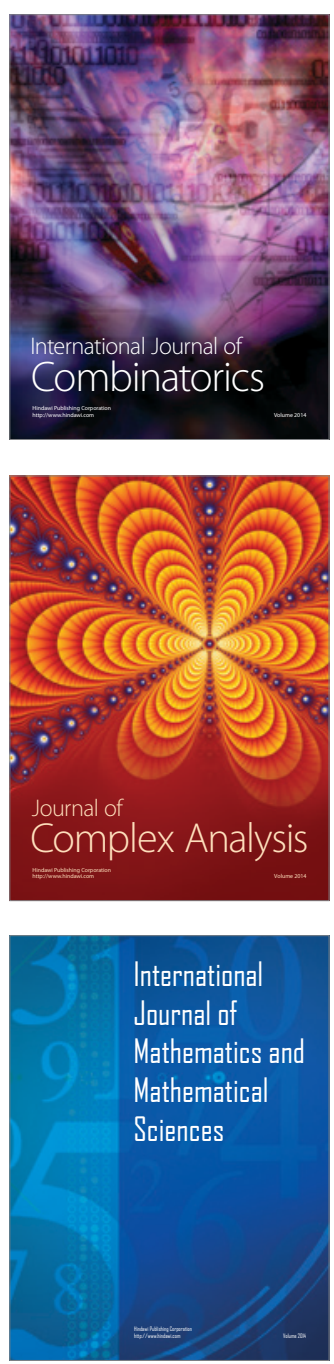
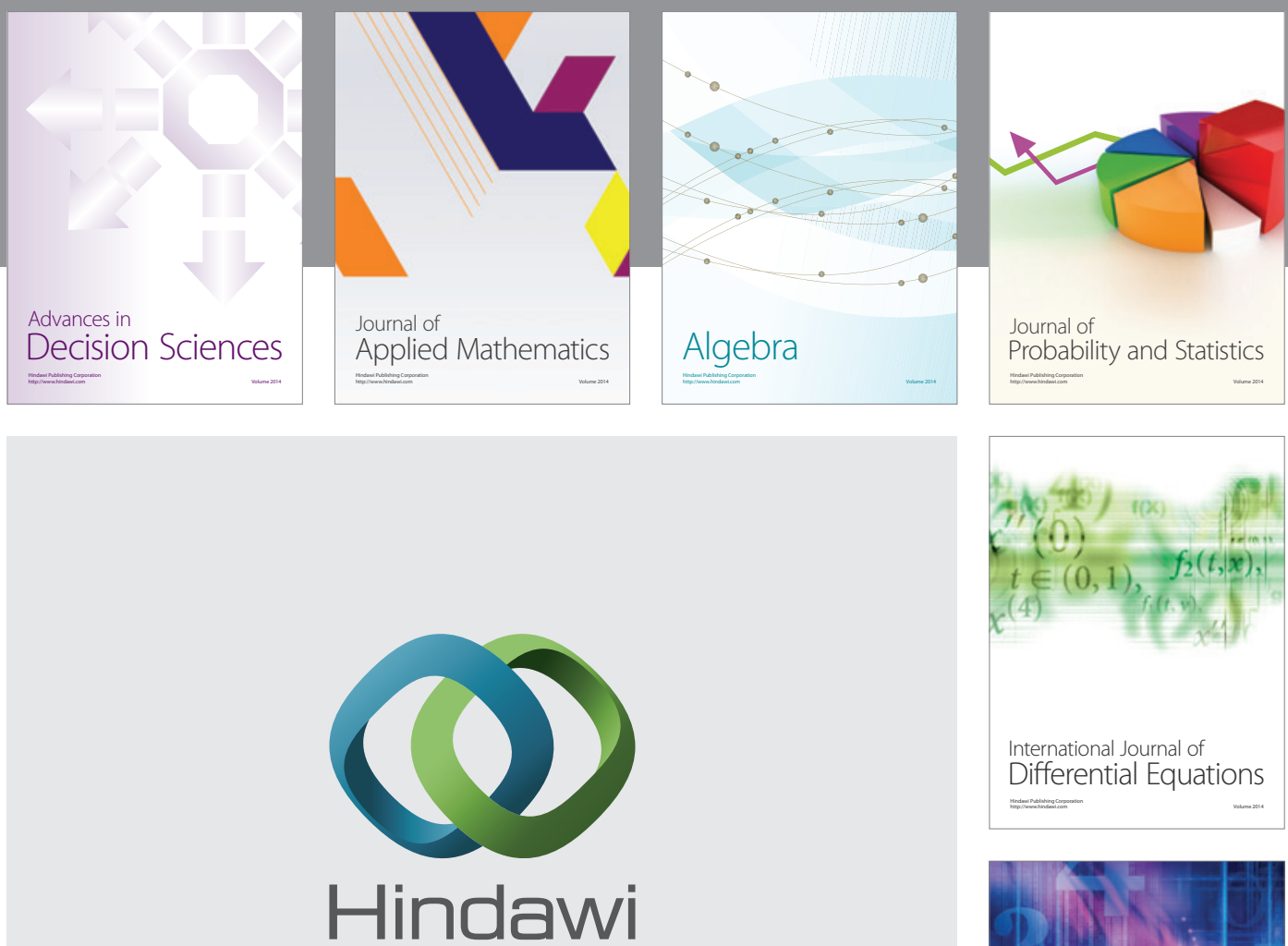

Submit your manuscripts at http://www.hindawi.com
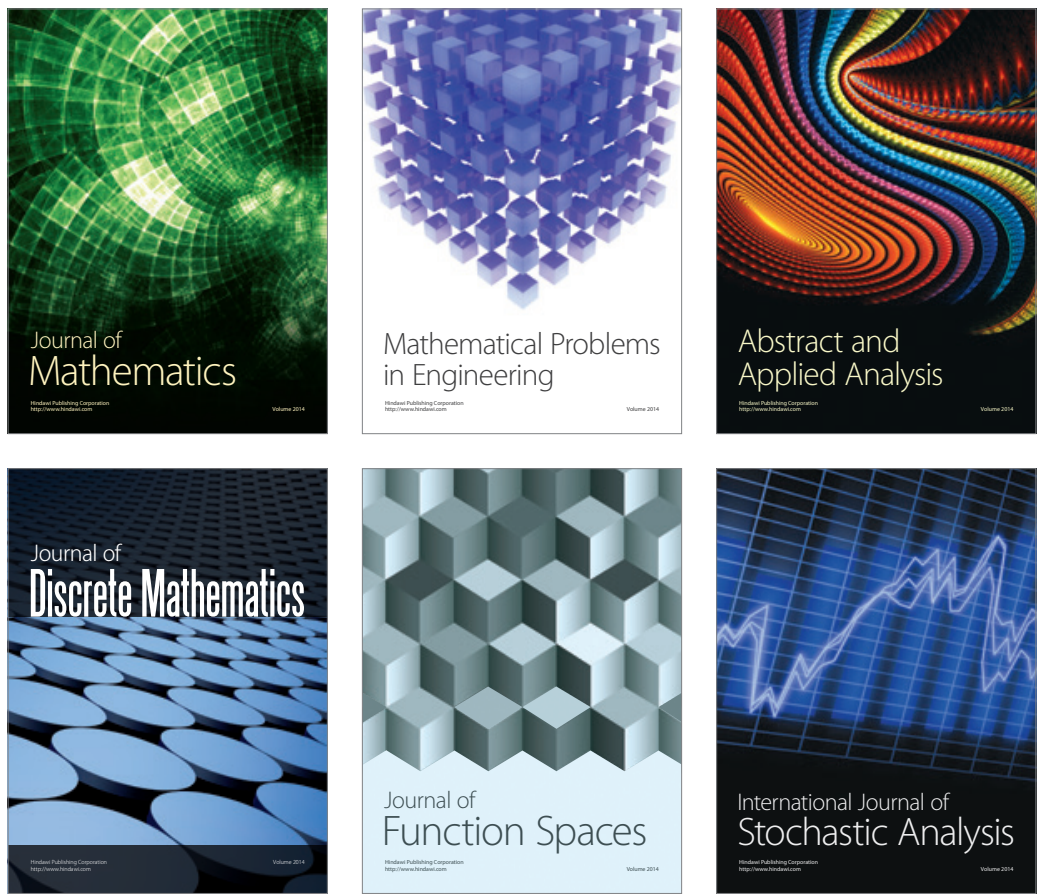

Journal of

Function Spaces

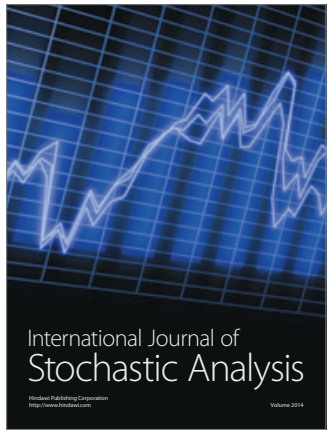

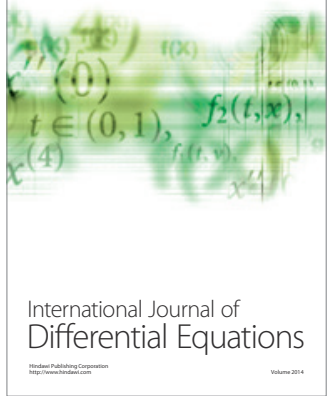
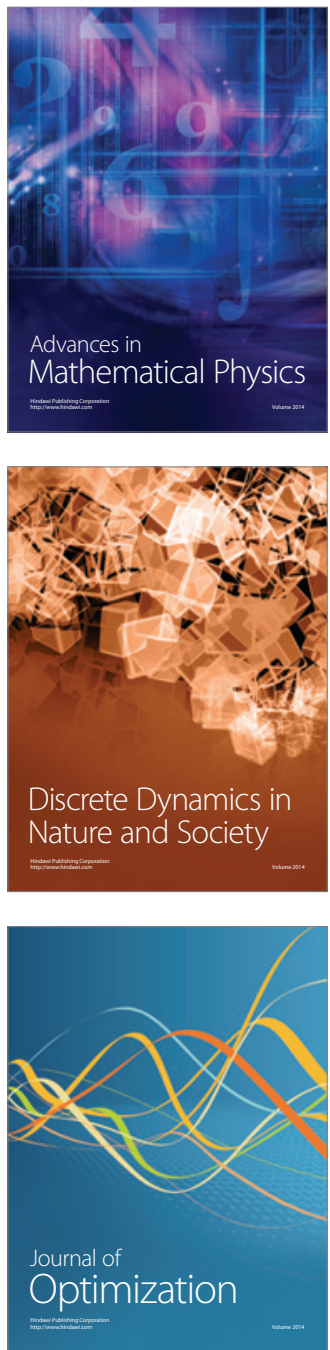